\title{
Sparübung des Bundesrates zulasten der ambulanten Versorgung
}

\section{Patrick Müller}

Leiter Abteilung Ambulante Versorgung und Tarife

\section{Die Vernehmlassung zum zweiten Eingriff des Bundesrates in den TARMED ist be-} endet. Das Bundesamt für Gesundheit entscheidet in den nächsten Wochen, wie die definitive Verordnung aussehen wird. Eines ist bereits sicher: Sollten die Massnahmen unverändert definitiv per 1. Januar 2018 in Kraft treten, wird die ambulante Versorgung massiv geschwächt. Damit werden letztlich die Patientinnen und Patienten die Verlierer sein!

Die rund dreimonatige Vernehmlassungsphase zum zweiten und wesentlich umfangreicheren Tarifeingriff des Bundesrates wurde am 21. Juni 2017 beendet. Zahlreiche Organisationen haben von der Möglichkeit Gebrauch gemacht, dem Bundesamt für Gesundheit BAG eine Stellungnahme dazu einzureichen. Das Departement Ambulante Versorgung und Tarife der FMH hat in einer ersten Phase der Vernehmlassung den Schwerpunkt auf die Analyse und Simulation der Massnahmen des zweiten Tarifeingriffes gelegt. Die Analysen ermöglichen der FMH auf Stufe Tarifposition pro Facharztdisziplin die Auswirkungen der einzelnen Massnahmen detailliert zu beurteilen. Diese Analysen und Simulationen waren zur Abschätzung der Auswirkungen und damit auch der Konsequenzen für die ambulante Versorgung in der Schweiz nötig.

\section{Allgemeine Beurteilung}

Der Bundesrat hat seine subsidiäre Kompetenz mit dem Verordnungsentwurf nicht korrekt wahrgenommen, denn sein Eingriff in die Tarifstruktur TARMED ist nicht mit den Vorgaben des KVG vereinbar und damit rein politischer Natur. Die subsidiäre Kompetenz des Bundesrates dient dazu, eine fehlende Sachgerechtigkeit zu korrigieren und sollte die Tarifstruktur nicht noch mehr verzerren und verschlechtern. Der Tarifeingriff dient einzig und allein einer einseitigen Kostensenkung: Mit einer Wiederherstellung der Sachgerechtigkeit hat dies nichts zu tun. Bereits der erste Tarifeingriff des Bundesrates 2014 verletzte die gesetzliche Vorgabe einer Sachgerechtigkeit, wie das jüngste
Gerichtsurteil des kantonalen Schiedsgerichts Luzern vom 29.05.2017 ${ }^{1}$ festhält und damit der klagenden Partei Recht gibt. Ferner hält es in seinem Urteil fest, dass

Gerade im OP-Bereich ist der Operateur teilweise mit langen Wartezeiten konfrontiert, in welchen er keine anderen Leistungen erbringen kann.

auch ein Tarifeingriff des Bundesrates gemäss KVG sachgerecht sein muss.

Insbesondere kommt es bei vielen Massnahmen zu einer überproportionalen Absenkung der Ärztlichen Leistung (AL) gegenüber der Technischen Leistung (TL). Gerade die Spezialisierung des nicht-ärztlichen Personals hat in den letzten Jahren zu einem starken Kostenschub innerhalb der Arztpraxen und Spitäler geführt. Da aber seit 2004 keinerlei Ausgleich durch Anpassung

\section{Simulieren Sie die Auswirkungen} auf Ihr Leistungsspektrum!

Was bedeutet der Tarifeingriff des Bundesrates und die vorgeschlagenen Massnahmen für die eigene Praxis? Muss ich mit Einbussen rechnen? Diese und ähnliche Fragen erreichen das Departement Ambulante Versorgung und Tarife aktuell täglich. Die Simulationen mit dem Tool Volumis Online unterstützen alle FMH-Mitglieder bei der Beantwortung dieser Fragen und ermöglichen jeder Ärztin / jedem Arzt die Auswirkungen des Tarifeingriffs bezogen auf ihr/sein individuelles Leistungsspektrum zu simulieren.

Mehr Informationen finden Sie auf myFMH und unserer Webseite: www.fmh.ch $\rightarrow$ AmbulanteTarife $\rightarrow$ TARMEDTarif $\rightarrow$ TARMED Änderungen ab 1.1.2018 
der Tarife im Bereich der TL erfolgte, wird bereits heute diese Kostenzunahme durch die "Ärztliche Leistung» quersubventioniert. Zudem fehlen Massnahmen, welche zur Korrektur von zu tief bewerteten und nicht mehr sachgerechten Leistungen und damit zu einer Aufwertung der TARMED-Leistung führen würden, gänzlich. So zum Beispiel die Aufhebung der Halbierung des Kostensatzes der letzten 5 Minuten (Position 00.0030) oder die Einführung einer InkonvenienzPauschale während der Sprechstunde.

\section{Die Anwendung von Abrechnungslimitationen ist für die FMH äusserst problematisch.}

Die vorgeschlagenen Massnahmen führen insgesamt zu einer deutlichen Schwächung der ambulanten $\mathrm{Me}$ dizin, setzen keine Anreize für die Erbringung der Leistungen im deutlich günstigeren und effizienteren ambulanten Bereich und gefährden damit die bereits jetzt schon problematische ambulante Versorgung der Bevölkerung in der Peripherie, ausserhalb der Ballungszentren. Damit handelt der Bundesrat entgegen dem von der Politik festgelegten Grundsatz «ambulant vor stationär».

\section{Bemerkungen zu einzelnen Massnahmen des Tarifeingriffes}

Nachfolgend kommentiert die FMH die drei Massnahmen der Verordnung, welche aus Sicht der Gesamtärzteschaft die grössten Auswirkungen haben. Die detaillierte Stellungnahme der FMH an den Bundesrat und das BAG ist auf der FMH-Webseite aufgeschaltet: www.fmh.ch $\rightarrow$ Ambulante Tarife $\rightarrow$ TARMED Tarif $\rightarrow$ TARMED Änderungen ab 1.1.2018

\section{Abschaffung der "Quantitativen Dignitäten»}

Mit der "Quantitativen Dignität» soll eine Kompensation der Weiter- und Fortbildungszeit (insbesondere der damit verbundenen reduzierten Lebensarbeitszeit) pro Spezialität ermöglicht werden. Für die FMH ist es unbestritten, dass die Löhne von Assistenz- und Oberärzten seit Einführung des TARMED angestiegen sind und Assistenz- und Oberärzte seit 2005 dem Arbeitsgesetz unterstellt sind. Dennoch ist es für das Lebenseinkommen auch heute noch entscheidend, wie lange ein Arzt in seine Weiter- und Fortbildung investieren muss: Während für den Weiterbildungstitel «Praktischer Arzt» drei Weiterbildungsjahre aufgewendet werden müssen, sind es für einen Facharzttitel mindestens 5 oder 6 Jahre.
Die generelle Kürzung für alle Fachdisziplinen mit einem Dignitätsfaktor unter 1,0 ist für die FMH nicht nachvollziehbar, widerspricht dem Prinzip der Sachgerechtigkeit und bedeutet nichts anderes als eine eigentliche Entschädigungskürzung von 3,2\%.

Aus Sicht der FMH und der ihr angeschlossenen Dachverbände sind auch weiterhin Differenzierungen der "Quantitativen Dignität» vorzunehmen, da für die Erbringung vieler Leistungen im TARMED zudem weitere Weiterbildungstitel notwendig sind, oder aber es muss ein zweiter Facharzttitel erworben werden. Sollen spezialisierte Leistungen, für die es viel Erfahrung braucht, weiterhin erbracht werden, so müssen die durch längere Weiterbildungszeiten verursachten Unterschiede im "Lebenseinkommen» kompensiert werden können. Die FMH hat zu diesem Thema, gemeinsam mit den angeschlossenen Dachverbänden ein neues, sachgerechtes und betriebswirtschaftliches Konzept ausgearbeitet, welches im Rahmen des Projekt TARCO umgesetzt werden soll.

\section{Erhöhung der "Ärztlichen Produktivität» in den Operationssparten (OP-Sparten)}

Die «Ärztliche Produktivität» soll die Zeit kompensieren, in welcher ein Arzt im Rahmen seiner TARMED-relevanten Tätigkeiten nicht direkt tarifwirksam sein kann, d.h. keine TARMED-Tarifpositionen verrechnen kann. Gerade im OP-Bereich ist der Operateur teilweise mit langen Wartezeiten konfrontiert, in welchen er keine anderen Leistungen erbringen kann. Ausserdem sind in der heutigen Ärztlichen Produktivität Leistungen berücksichtigt, die nicht direkt dem Patienten verrechnet werden können, wie z.B. das «Sign In", «Sign Out», Wechselzeiten, Teambesprechungen oder Rapporte und Operationsvorbereitungen in Anwesenheit des Operateurs (Anästhesie und Lagerungen im Beisein des Operateurs). Entsprechend tief ist die Produktivität im OP-Saal. Eine Anhebung der ärztlichen Produktivität im OPSaal, wie der Bundesrat sie vorsieht, kann nur in Verbindung mit der einzelnen Tarifierung der präoperativen Massnahmen sowie der postoperativen Betreuung umgesetzt werden. Dies ist im Projekt TARCO so vorgesehen. Bevor aber diese Faktoren, welche heute nicht im Tarif abgebildet sind, berücksichtigt werden können, muss die "Ärztliche Produktivität» für den OPBereich wieder auf das ursprüngliche Niveau abgesenkt werden. Ohne dass die vom Operateur zusätzlich erbrachten Leistungen, die bisher über die tiefere Produktivität entschädigt wurden, in der Tarifstruktur berücksichtigt werden, ist eine Erhöhung weder sachgerecht noch betriebswirtschaftlich. 


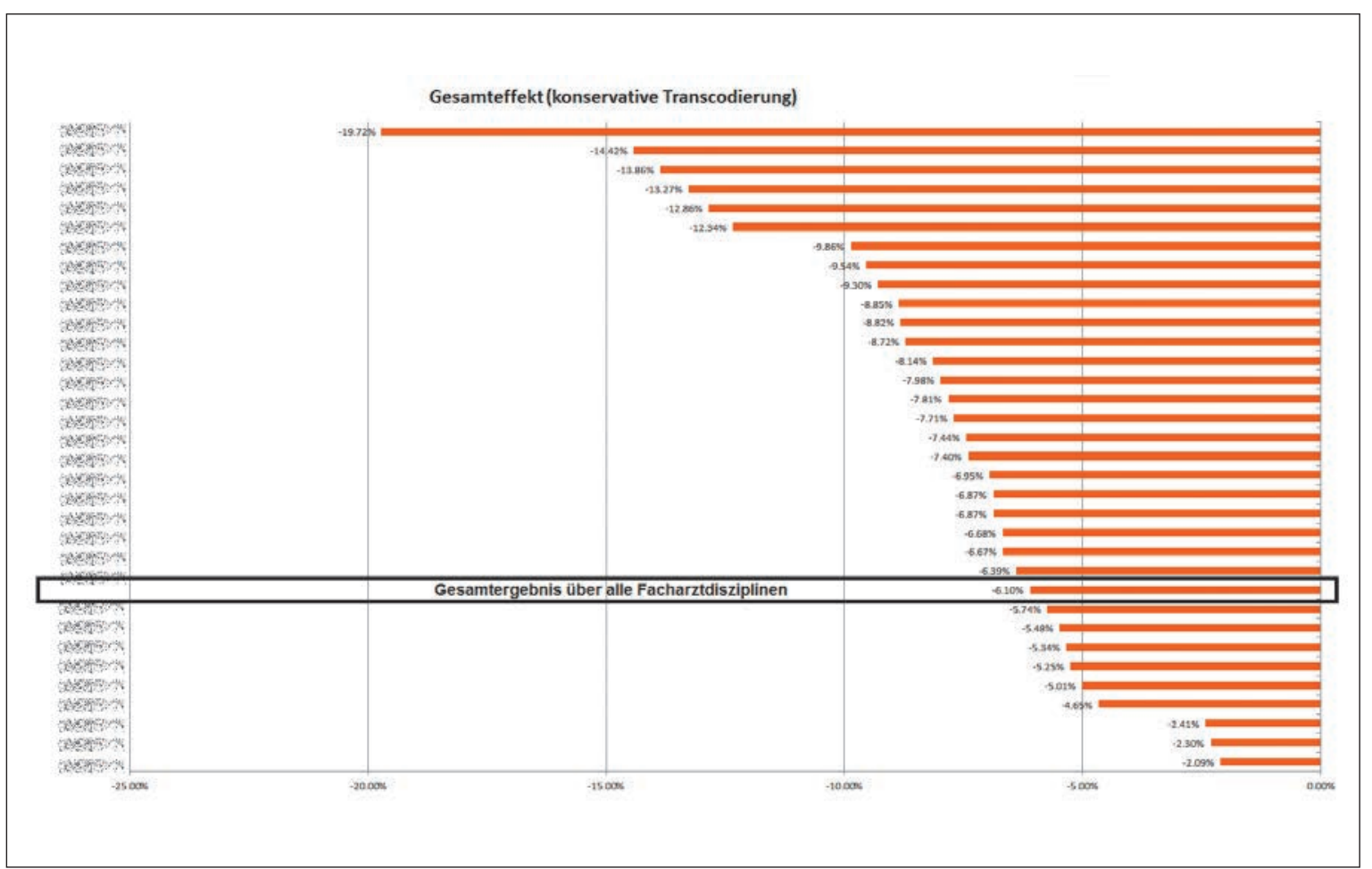

Sparübung des Bundesrates: Gesamteffekt der Auswirkungen des zweiten Tarifeingriffs pro Facharztdisziplin (Total Taxpunkte).

\footnotetext{
$2 \mathrm{http://www.interpharma.}$ $\mathrm{ch} /$ fakten-

statistiken/1870-pra emien-steigen-staerkerals-die-gesundheitskosten 12. Mai 2017
}

\section{Anwendung von Abrechnungs- limitationen}

Die Anwendung von Abrechnungslimitationen ist für die FMH äusserst problematisch. Mit der Einführung weiterer Limitationen insbesondere auf den ärztlichen Grundleistungen wie Konsultation und ärztlichen Leistung in Abwesenheit wird eine verdeckte Rationierung eingeführt. Gerade bei Kindern und Jugendlichen, älteren Menschen oder Menschen mit psychischen Erkrankungen sind Gespräche in Abwesenheit des Patienten mit Angehörigen, anderen Medizinalund Gesundheitsfachpersonen und Behörden ein wichtiger und unerlässlicher Bestandteil der ärztlichen Behandlung. Mit diesen umfassenden Limitationen wird ein falscher Anreiz gesetzt. Damit ist der Patient letztlich der Leidtragende, denn interprofessionelle und interdisziplinäre Arbeit für den Patienten wird erschwert.

Die FMH fordert den Bundesrat nachdrücklich auf, die Limitationen im Einzelfall mit den entsprechenden Experten der Fachgesellschaften und FMH zu prüfen und zu überdenken.

Die FMH hat die Effekte pro Massnahme und den Gesamteffekt des zweiten Tarifeingriffes bezogen auf die einzelnen Facharztdisziplinen analysiert und aufgrund einer eigens erstellten Transcodierung die Auswirkungen simuliert. In der beiliegenden Grafik sind die Auswirkungen bezogen auf die Facharztdisziplinen zu entnehmen. Dabei entspricht ein Balken einer Fachdisziplin. Aufgrund von Datenschutzvorgaben sind die einzelnen Disziplinen unkenntlich dargestellt. Eines wird klar: Vom Tarifeingriff sind alle betroffen!

\section{OKP-Prämien durch eine einheitliche Finanzierung stabilisieren}

Mit jedem Tarifeingriff verändern sich auch die Anreize. In vorliegendem Fall ist mit einer Verlagerung von Leistungen, die ambulant nicht mehr kostendeckend erbracht werden können, in den stationären Bereich zu rechnen. Ist dies allenfalls vom Bundesrat

\section{Mit jedem Tarifeingriff verändern sich auch die Anreize.}

gewünscht, damit eine vordergründige Entlastung der OKP-Prämien erreicht werden kann? Die OKP-Prämien sind in den letzten 20 Jahren um fast 50\% stärker gestiegen als die Gesundheitskosten ${ }^{2}$. Diese ungleiche Entwicklung erfolgte aufgrund der unterschiedlichen Finanzierungsmodelle: Während die Finanzierung der stationären Versorgung dualfix (durch Kantone/Versicherer) organisiert ist, wird die ambulante Versorgung 


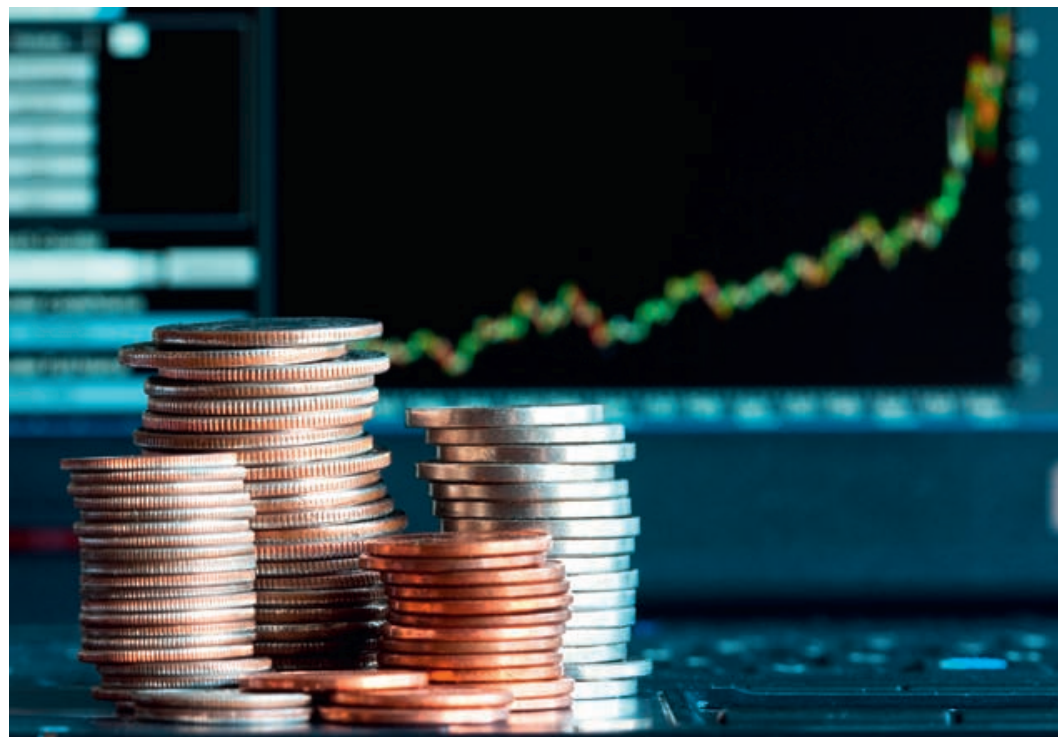

Die OKP-Prämien sind in den letzten 20 Jahren um fast $50 \%$ stärker gestiegen als die Gesundheitskosten.

Korrespondenz: FMH / Abteilung Ambulante Versorgung und Tarife Baslerstrasse 47

CH-4600 Olten

Tel. 0313591230

Fax 0313591238

tarife.ambulant[at]fmh.ch monistisch (nur durch Versicherer zu Lasten der OKPPrämien) finanziert. Der Tarifeingriff gefährdet die angestrebte und ausdrücklich gewollte Verschiebung von der stationären, hin zur kostengünstigeren und volkswirt-schaftlich sinnvolleren ambulanten Versorgung und widerspricht damit auch der Strategie Gesundheit2020 des Bundesrates. Um den jährlichen Prämienanstieg zu dämpfen, erfolgt durch die Politik einmal mehr eine Symptombekämpfung, anstatt dass Fehlanreize im System eliminiert würden.

\section{Wiederherstellung der Sachgerechtig- keit ist nur durch eine Gesamtrevision möglich}

Mit diesem zweiten, sehr umfassenden Tarifeingriff des Bundesrates wird die Bereitschaft der Krankenversicherungsverbände, an einer Gesamtrevision aktiv und engagiert mitzuarbeiten, nicht grösser. Denn die
Sparübung des Bundesrates verspricht eine Prämienentlastung von 700 Millionen Franken womit auch das OKP-Vergleichsvolumen bei den Verhandlungen der Gesamtrevision sinkt. Für die FMH und die in der Ärztekammer vertretenen Organisationen ist der Revisionsbedarf der 2004 eingeführten ambulanten Tarifstruktur TARMED unbestritten. Nach der Ablehnung des ersten Revisionsvorschlages in der Urabstimmung unter allen Mitgliedern im Mai 2016 hat die FMH deshalb das Revisionsprojekt TARCO zur Nachbesserung gestartet. TARCO bezweckt eine sachgerechte und betriebswirtschaftliche Tarifierung aller ambulanten ärztlichen Leistungen. Denn nur eine umfassende Tarifrevision gemeinsam mit allen Ärzteorganisationen und Tarifpartnern ist aus Sicht der Ärzteschaft der einzig richtige Weg. Die FMH verfolgt das Projekt TARCO weiterhin mit grossem Engagement.

\section{Ausblick}

In den nächsten Wochen wird der Bundesrat die definitive Verordnung und damit auch die definitiven Massnahmen, welche per 1. Januar 2018 in Kraft treten sollen, bekannt geben. Aufgrund der umfassenden Änderungen, die jede Ärztin und jeden Arzt treffen werden, ist nicht nur eine Umstellung oder Anpassung der Leistungserfassungs- und Abrechnungssoftware notwendig, sondern auch eine Überprüfung der Sprechstunden- oder Behandlungszeiten infolge neuer Limitationen unerlässlich. Die der FMH angeschlossenen Ärzteorganisationen und FMH-Einzelmitglieder werden mit gezielten Informationen, häufig gestellten Fragen (FAQ), Factsheets, Tarifbrowser und Publikationen auf die Einführung vorbereitet. Die FMH wird nach Bekanntgabe der definitiven Verordnung erneut informieren.

\section{Bildnachweise}

Grafik: Transcodierung FMH, basierend auf Daten NewIndex, April 2017 Bild: @ Kenishirotie | Dreamstime.com 